\begin{tabular}{|c|c|c|}
\hline Beitr. Ent. & Keltern & ISSN 0005-805X \\
\hline $\mathbf{5 7}(2007) 1$ & S. $161-175$ & 30.06 .2007 \\
\hline
\end{tabular}

\title{
Neue Aspekte bei der Einlagerung von Beute durch die solitäre Wespe Symmorphus crassicornis (PANZER) in ihre Brutzellen
}

\author{
(Hymenoptera, Vespidae, Eumeninae)
}

Mit 9 Figuren

Arnim Tölke

Meiner Ehefrau Ingeborg Tölke zu ihrem 80. Geburtstag am 28. Januar 2007 in Dankbarkeit gewidmet. Sie nahm in den vergangenen vier Jahrzehnten meiner wissenschaftlichen Tätigkeit daran nicht nur verständnisvollen Anteil, sondern wirkte in vielerlei Hinsicht bei all diesen Vorhaben auch mit.

\section{Zusammenfassung}

Dem Autor gelang es, Symmorphus crassicornis (PANZER) in ein Bambus-Brutrohr zu locken, welches im Mittelteil ein $5 \mathrm{~cm}$ langes Stück Acrylrohr eingesetzt bekommen hatte. Durch diese nun mögliche Sichtbeobachtung konnte er nachweisen, dass die solitäre Wespe in der Regel nicht sofort ihre Beute in die Brutzelle einsortiert. Vielmehr fängt sie eine bestimmte Menge an Blattwespenlarven und legt sie innerhalb des Brutrohres vor der Brutzelle erst einmal ab. Einen Kontakt mit dem an der Decke der Brutzelle an einem Faden hängenden Ei erfolgte in dieser Einlagerungsphase in keinem Fall. Die gefangenen Larven deponiert sie in dieser Phase entweder einzeln hintereinander oder wenn sie eine geringe Größe besitzen auch einmal nebeneinander, in keinem Fall aber übereinander. Erst dann, wenn die Wespe eine bestimmte Anzahl an Beute so in den Vorraum eingetragen hat, beginnt sie mit dem Einsortieren in die Brutzelle. Dabei packt sie diese mit den Mandibeln und zieht sie rückwärtsgehend in die Brutzelle hinein. Hierbei wählt sie immer die größte Larve zuerst, dann die Zweitgrößte usw. Bemerkenswert ist auch, dass sie die im Vorraum liegenden Larven unabhängig vom Füllgrad der Brutzelle in jedem Fall alle einzieht. Es bleibt somit keine Einzige außerhalb der Brutzelle liegen. Wenn beim hineinziehen Schwierigkeiten eintreten, geht sie zum Schieben über. Ist die Brutzelle sehr voll gefüllt, zieht sie hin und wieder eine Larve heraus und versucht es mit einer andersartigen Einsortierung. Der schon zu früheren Zeiten bei Symmorphus crassicornis (PANZer) nachgewiesene Eikontakt (TölKe, A. 1996), der dadurch erfolgt, dass die Wespe mit einem ihrer Fühler gegen das frei hängende Ei schlägt und durch die so erzeugte Schwingung einen Pendelrückschlag bewirkt, den sie dann mit dem gleichen Fühler wieder abfängt, spielt in der Steuerung der einzusortierenden Beute eine völlig andere Rolle, als bisher vermutet. Denn empfängt die Wespe auf Grund des Füllungsgrades nach Einsortieren der letzten im Vorraum abgelegten Larve nach ihrem Fühlerschlag gegen das Ei keinen EiRückschlag, schließt sie die Brutzelle mit Lehm. Aber auch dann, wenn ein Schlag gegen das Ei auf Grund einer besonderen Situation nicht möglich ist, verschließt sie ebenfalls diese Brutzelle nach Einsortierung aller im Vorraum liegenden Larven. Dieser Nachweis gelang dem Autor auch indirekt mit einem Experiment dadurch, dass ein von der Wespe besetztes Bambusrohr bei jeder neu angelegten Brutzelle er es um jeweils 180 Grad drehte, nachdem das Ei gelegt war. Als Folge davon lag das Ei somit am Boden der Brutzelle und ein Pendelschlag war unmöglich. Aber auch unter solchen Bedingungen erfolgte kein sofortiges Schließen der Zelle, sondern auch hier erst, nachdem alle im Vorraum abgelegten Blattwespenlarven in die Brutzelle einsortiert waren. Führt aber die Wespe unter Normalbedingungen einer Einsortierung den schon beschrie- 
benen Pendelschlag aus und empfängt sie auch den Pendelrückschlag, weil noch Raum zwischen Ei und einsortierten Larven vorhanden ist, beginnt sie noch einmal mit der Jagdphase und legt wiederum zuerst einmal ihre gefangene Beute im Brutrohr vor der Brutzelle ab und sortiert schließlich auch diese Larven in die Brutzelle.

\section{Summary ${ }^{1}$}

The author managed to lure Symmorphus crassicornis (PANZER) into a bamboo nest tube, the central part of which had been replaced by a $5 \mathrm{~cm}$ long acrylic tube. Visual observation made possible in this way enabled the author to find out that the solitary wasp does not usually sort its prey immediately into the brood cell. Rather, it catches a certain quantity of sawfly larvae and deposits them first in front of the brood cell inside the breeding tube. The egg, suspended on a filament hanging down from the ceiling of the brood cell, is not being contacted in any case. In this phase the wasp deposits the caught larves either individually one behind the other or even occasionally - when these are of a certain size - one alongside the other, but never one upon the other. The wasp does not start sorting the prey into the brood cell until it has carried a certain quantity of prey to the space in front of it. In doing so, it takes the prey with its mandibles and pulls it, walking backward, into the brood cell. It always chooses the largest caterpillar first, then the second largest, etc. It is also worth mentioning that the wasp always removes all caterpillars lying in front of the brood cell irrespectively of how full the brood cell actually is. That means that not a single item of prey remains outside the brood cell. If the wasp has difficulty pulling the prey, it switches from pulling to pushing. If the brood cell is very full, it occasionally happens that the wasp removes a caterpillar from it and tries a different kind of sorting. The egg contact proved before (TöLKE, A. 1996) in respect of Symmorphus crassicornis (PANZER), which happens so that the wasp uses one of its antennae to strike against the freely suspended egg, making it oscillate so that it effects a pendulum-type rebound, which the wasp intercepts using the same antenna, plays a completely different role than has been assumed so far in controlling the prey to be sorted into the nest. For if, due to a high filling level reached after the last deposited caterpillar was sorted into the nest, the wasp does not feel the egg rebound, it closes the brood cell with loam. But even if the wasp cannot strike against the egg because of particular circumstances, notwithstanding it closes this brood cell, when all caterpillars in front of it have been sorted into the nest. The author managed to prove this behaviour also indirectly by way of an experiment, rotating a bamboo tube, occupied by a wasp, 180 degrees each time a new brood cell had been built and the egg had been laid. As a consequence, the egg lay on the floor of the brood cell and a strike causing the pendulum-type rebound was not possible. But even under such circumstances the cell was not closed immediately, but, as usually, only after all sawfly larvae deposited in the front area of the nest had been sorted into the brood cell. However, if a prey-sorting wasp under normal nesting conditions effects the pendulum-type strike described above and also feels the rebound, it once again proceeds to the hunting phase and, as a first step, deposits the prey it has caught in the breeding tube in front of the brood cell, as described, and finally sorts these larves into the brood cell as well, as described above.

\section{Резюме}

Автору удалось заманить осу Symmorphus crassicornis (PANzeR) в труббк-инкубатор из ствола бамбука, среднюю часть которого заменили куском акриловой трубки Алиной в 5 см. Благодаря созданной возможности визуального наблюдения автор сумел доказывать, что одиночная оса, как правило, сразу не укладывает свою добычу в «инкубатор». Скорее, она мовит определенное количество Аичинок пилильщика и сначала укладывает их внутри трубки перед «инкубатором». В этой фазе, т.е. во время укладки добычи, ни в коем случае не имело места контакта с яйцом, которое висело на нитке с потолка «инкубатора». В этой фазе оса укцадывает пойманных гусениц или одну за Аругой или, если они небольшие по размеру, также одну возме Аругой, однако ни в коем случае одну наА Аругой. УАожив определенное количество добычи в описанном виде перед «инкубатором», оса начинает сортировать (перекладывать) Аобычу в него. При этом она захватывает Аобычу мандибулами и,

\footnotetext{
${ }^{1}$ Die englische und russische Übersetzung fertigte Peter Zaumseil. Dafür danke ich ihm.
} 
идя задом, тащит ее в «инкубатор». Первой она всегда выбирает самую большую гусеницу, потом вторую по размеру и т.А. Аостойным упоминания является тот факт, что она всегАа втягивает всех гусениц, Аежащих перед «инкубатором», независимо от степени его наполнения. Таким образом, вне «инкубатора» не остается ни одной гусеницы. Если у осы возникают трудности тащить добычу, она заталкивает ее. Бывает, что при очень наполненном «инкубаторе» оса вытягивает одну из гусениц и пытается уложить ее по-другому. Контакт с яйцом - факт, уже Аоказанный раньше в отношении осы Symmorphus crassicornis (PANZER) (Тёльке, А. 1996 г.), причем контакт устанавливается тем, что оса ударяет одним из щупалец по свободно висящему яйцу и тем самим приводит его в колеблющееся Авижение, которое она перехватывает тем же щупацьцем, когда яйцо возвращается обратно. Этот контакт имеет отличное значение Аля интерпретации поведения осы относительно сортировки Аобычи по сравнению с тем, что предполагали до сих пор. Это поведение объяснялось тем, что оса, переложив последнюю гусеницу из трубки в «инкубатор», запечатывает «инкубатор» глиной, если она больше не ощущает обратный удар от колеблющегося яйца. ОАнако после того как все гусеницы, межавшие в трубке, были переложены в «инкубатор», оса запечатывает «инкубатор» Ааже в том случае, если из-за некоторой сложившейся ситуации ей невозможно уАарять по яйцу. Автору удалось косвенно Аоказывать это также с помощью эксперимента, а именно тем, что поворачивац заселенную осой трубку из ствола бамбука в 180 граАусов каждый раз, когАа оса отложила яйцо. Вследствие этого, яйцо межало на Ане «инкубатора», а удар, вызывающий колеблющееся движение, был невозможным. ОАнако и в этих условиях оса запечатывает «инкубатор» не сразу, а только после того, как она переложила туда всех мичинок пилильщика, накопленных перед «инкубатором». Но в нормальных условиях, выполнив описанный выше удар, вызывающий колеблющееся движение яйца и ощутив обратный толчок от этого Авижения - поскольку было еще Аостаточно большое пространство между яйцом и подлежащими сортировке гусеницами - оса еще раз вступает в фазу охоты и опять сначала укладывает пойманную добычу в трубку переА «инкубатором» и в конце сортирует гусениц в «инкубатор», как описано выше.

\section{Einleitung und Rückblick}

In einer Untersuchung des Autors, die 4 Jahrzehnte zurück liegt (nicht veröffentlichte Diplomarbeit, Tölke, A. 1966), blieben im Brutverhalten der solitären Wespe Symmorphus crassicornis (PANZER) einige sehr wesentliche Fragen ungeklärt. Seinerzeit wurde zwar von ihm aus der sekundengenauen Registrierung der Ein- und Ausflugzeiten ein Eikontakt postuliert, aber er konnte ein solches Verhalten nicht beweisen. TöLKE, I. (1968) gelang es mit Hilfe einer $16 \mathrm{~mm}$ Filmkamera (Pentaflex 16) einen in der Brutzelle erfolgenden Eikontakt erfolgreich zu filmen, indem sie eine Brutzelle mit einer Mikroskopleuchte von außen her ausleuchtete und das Verhalten im Innern einer Brutröhre filmisch erfasste, was bei der Einlagerung einer Blattwespenlarve in der Brutzelle ablief. In dieser Filmszene war das an der Decke an einem feinen Faden hängende Ei (Fig. 1) deutlich sichtbar.

Die Wespe schlug mit einer Antenne gegen das Ei, das dadurch einen Pendelausschlag ausführte, deren Gegenschlag wiederum von der gleichen Antenne aufgefangen wurde. Damit war ein durch

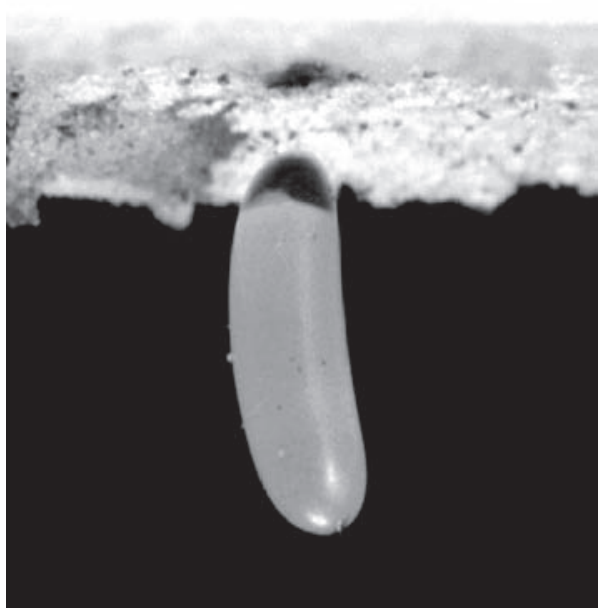

Fig. 1: Das Ei hängt an einem feinen Faden oben an der Decke der Brutzelle. 
die Wespe ausgeführter Eikontakt nachgewiesen. Offen blieb aber hierbei die Frage, ob die weitere Einlagerung von Larven von einem zwischen dem unteren Eipol und den Larven indirekt ermittelten freien Raum abhängt. Oder anders gesagt, ob mit Hilfe des Eikontaktes die Wespe einen nicht mehr vorhandenen freien Raum erkennt, welcher dann in dieser Situation eine beendende Bedingung auslösen könnte. Auf jeden Fall verschließt die Wespe die Brutzelle mit Lehm, wenn eine nicht bekannte Endbedingung vorliegt. Auch wenn die Postulierung einer solchen Endbedingung für den Ablauf einer Einlagerung von Blattwespenlarven eine hohe Wahrscheinlichkeit besaß, der endgültige Beweis konnte aus der Filmsequenz nicht abgeleitet werden.

In einer an dem Wohnhaus des Autors befindlichen Wildbienen-Anlage legte im Jahre 2000 ganz überraschend nach langer Zeit wieder einmal eine solitäre Wespe der Art Symmorphus crassicornis (PAnZer) Brutzellen in einer Bambusröhre an. Das Besondere bestand diesmal darin, dass die solitäre Wespe ein Bambusrohr dafür nutzte, das im Röhrendurchmesser für diese Art erstaunlich groß war und eine Ausleuchtung wie durch I. TöLKE praktiziert, jetzt endlich wieder einmal ermöglichte. Zusätzlich ungewöhnlich günstig war für eine exakte Beurteilung des Verhaltensablaufs die Möglichkeit, den jeweiligen Beginn der Schließung der Brutzelle mit Lehm genau bestimmen zu können, weil die Wespe den Lehm von einem unterhalb der Brutröhre auf einem Brett befindlichen Lehmhaufen holte. Hier konnte sie bequem beobachtet werden. Dadurch gelang es, die nach dem Verschließen der Brutzelle fast immer unmittelbar folgende Eiablage in ihrem zeitlichen Umfang genau zu bestimmen und dieses Verhalten zusätzlich als Videosequenz zu filmen. Zusätzlich gelang dem Autor durch diese außergewöhnliche Situation, nach dem Eintrag von Larven diesen schon beschriebenen Eikontakt mit einem Video-Camcorder bei zwei Einsortierungen zu filmen und dadurch ebenfalls zweifelsfrei nachzuweisen.

In den vergangenen Jahren hat es von Seiten des Autors nicht an Versuchen gefehlt, diesen Pendelschlag gegen das Ei bei der Einlagerung von Beute zu interpretieren. Denn es lag nahe anzunehmen, dass damit die Wespe indirekt die Menge der einzutragenden Blattwespenlarven steuert. Siehe hierzu Tölke, A. (1996). In dieser nun hier vorliegenden Arbeit werden bisher unbewiesene Verhaltensweisen im Brutverhalten dieser Art geklärt.

Durch den fehlenden Beweis, ob die Wespe die Einlagerungsmenge an Larven tatsächlich mit dem Schlag gegen das hängende Ei steuert, versuchte der Autor in der Vergangenheit dies durch sekundengenaue Registrierung ihrer Aktivitätszeiten bei den Ein- und Ausflügen zu klären. Hierbei half ein 16 Bit-Home Computer (Texas Instruments TI-99/4A), später ein auf dem PC laufender Emulator (PC 99) mittels eines selbst geschriebenem Extended Basic-Programms das im Innern der Brutröhren ablaufende Verhalten zu erkennen (TöLKe, A. 2005). Während dies bei der solitären Biene, Osmia rufa (L.), letztlich mühelos gelang (TöLKE, A. 1973), gab es bei dieser Wespenart durch unerwartete Hindernisse kein Endergebnis. Der Grund für diese Schwierigkeiten lag darin begründet, dass jedes Individuum dieser Art zwar ein festes zeitliches Grundverhalten besitzt, aber in einigen Verhaltensabschnitten seine „zeitliche individuelle Note“ ablaufen lässt und damit von den bekannten Standardwerten abweicht.

Bei den nachträglichen Berechnungen der jeweiligen Aufenthaltzeit und den dann folgenden Analysen fiel bei jedem Individuum gewissermaßen immer wieder auf, dass beim Einbringen der Beute die Wespe über mehrere Wiederholungsschritte relativ kurze Aufenthaltzeiten benötigt. Die diesbezügliche auftretende Bandbreite, die eine Wespe zeitlich gesehen für jeden Aufenthalt in der Brutröhre hierbei in Anspruch nimmt, zeigt aber von Individuum zu Individuum durchaus unterschiedliche Werte. Manche Wespen benötigen hierfür nur Zeiten zwischen 10 bis 20 Sekunden. Bei anderen Wespen liegt der zeitliche Spielraum sogar zwischen 40 bis 60 Sekunden. Der schon vorhandene Teil des Computerprogramms, der das Einbringen von Beute aus den Aktivitätszeiten interpretiert, ist aber so formuliert, dass sich diese Bandbreite der absoluten Einlagerungszeit sich 
nicht quantitativ, wohl aber qualitativ auswirkt. Also Verhaltensphase für Verhaltensphase in jedem Fall mühelos vom Computer als Einbringen von Beute aus den Aktivitätszeiten richtig erkennt. Die Gründe für die quantitativen Abweichungen der Einlagerungszeiten könnten ihre Ursache im unterschiedlichen Durchmesser der Röhre (vielleicht je größer desto schneller) liegen oder zusätzlich von der Größe der eingebrachten Blattwespenlarve abhängen. Aber unabhängig von quantitativen Abweichungen fällt in jedem Fall auf, dass die Einbringungszeiten im Verhältnis zu anderen Aktivitätszeiten immer relativ kurz sind. Aus alldem leitete der Autor die Arbeitshypothese ab, dass die Wespe die gefangene Beute nicht sofort in die Brutzelle einsortiert, sondern sie mit hoher Wahrscheinlichkeit irgendwo im Brutrohr lediglich vorübergehend ablegt. Und dann spräche sehr viel dafür, dass dies nicht schon innerhalb der aktuell vorgesehenen Brutzelle sondern davor geschieht. Denn in dieser kurzen Zeit des Einbringens wäre anderes kaum vorstellbar. Nach dem Einbringen der 4. bis 6. Larve tritt dann immer eine sehr lange Aufenthaltszeit auf. Da dieses Verhalten bei jeder Wespe dieser Art zu beobachten war, schloss daraus der Autor, dass sie erst dann, wenn eine bestimmte Larvenmenge im Rohr vor der Brutzelle liegt, mit dem eigentlichen Einsortieren der Larven in die Brutzelle beginnt. Für diese lange Zeitverhaltensphase wurde schon 1966 von ihm ein Sortieren postuliert und diese Phase erhielt deshalb vom Autor den Namen „Sortierphase“. Bei entsprechendem Rohrdurchmesser folgt in manchen Fällen sogar nach der ersten Sortierphase noch einmal das Eintragen von weiteren 4 bis 5 Larven. Auch diese Phase schließt die Wespe mit einer „Sortierphase“ ab. Endgültige Beweise konnte der Autor für ein derartiges Verhalten für diese Wespenart damals aber nicht erbringen. Auch diese Fragen sollten mit einer erneuten Untersuchung nach Möglichkeit einer Klärung zugeführt werden.

\section{Material und Methode}

Es galt Methoden zu finden, mit deren Hilfe die hier im Brutverhalten von Symmorphus crassicornis (PANZER) angesprochenen noch offenen Fragen geklärt werden können.

\subsection{Gezielte Experimente mittels Veränderung der Lageposition des Eies innerhalb der Brutzelle}

Wenn dem an der Decke an einem feinen Faden hängenden Ei durch eine zeitweilig erzeugte Schwingung beim Schlag durch einen Fühler der Wespe eine zentrale Rolle bei der Begrenzung der Beutemenge zukommt, dann müsste durch eine Veränderung der Lageposition des Eies die Einlagerung von außen beeinflußbar sein. Es war deshalb zu erkunden, was geschieht, wenn das Brutrohr um 180 Grad verdreht würde und damit das Ei nicht mehr schwingen kann, weil es am Boden der Brutzelle zum Liegen kommt. Diese Methode der Veränderung der Positionierung des Eis erfolgte im Jahr 2000 in einem von der Wespe belegten Bambusrohr immer unmittelbar danach, wenn die Wespe ein Ei an die Decke geheftet hatte. Um Zufälle nach Möglichkeit auszuschließen, erfolgte diese Verdrehung bei jeder neu angelegten Brutzelle innerhalb des Brutrohres. Zur Kontrolle geschah dies bei einer Brutzelle aber nicht, weil die Registrierung der Beuteeinlagerung der ersten Brutzelle erkennen ließ, dass in diesem Brutrohr im Normalfall nach der ersten Sortierphase noch eine zweite folgt. Somit im ungestörten Brutablauf jede Brutzelle ca. 10 bis 12 Blattwespenlarven aufnahm.

\subsection{Ausgelegte Röhren oder spezielle Holzbohrungen, die sich öffnen lassen}

Um nach dem Einbringen von Larven beurteilen zu können, wohin sie innerhalb des Rohres abgelegt würden, kamen speziell präparierte Bambusröhren bzw. angebohrte Holzblöcke zum 
Einsatz. Im einfachsten Fall wurde ein Bambusstück in der Länge gespalten und dann mittels Wäscheklammer wieder zusammengefügt. So wäre es möglich gewesen, nach Ausflug der Wespe kurz einmal in das Innere des Rohres zu schauen, ohne sie in ihrem Brutverhalten allzusehr zu stören. Nach dem gleichen Prinzip geschah die Präparation eines Holzblockes, der in der Länge lang aufgetrennt wurde. An der Trennstelle erfolgten die Bohrungen (Fig. 2).

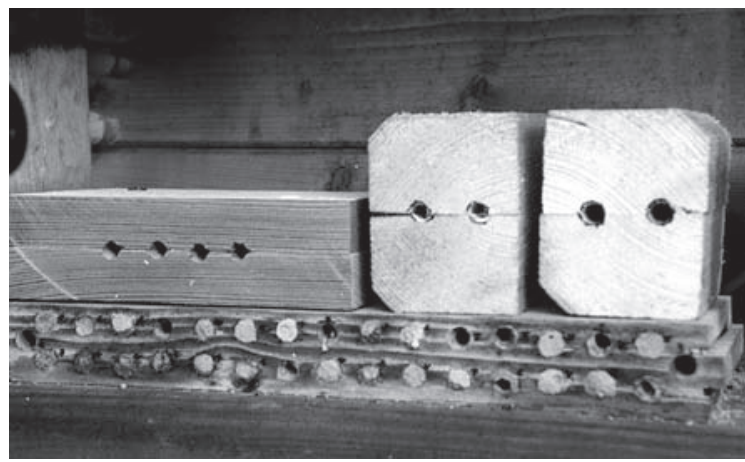

Fig. 2: Speziell präparierte Holzblöcke, die aber von keiner Wespe angenommen wurden.
Mittels zweier Holzschrauben wurden diese zwei Teile wieder zu einem Stück verschraubt. Auch hier wäre es möglich gewesen, jederzeit bei vorsichtigem Öffnen des Holzstückes durch Sichtkontrolle zu beurteilen, wo die Larven innerhalb der Röhren zu einem bestimmten Zeitpunkt vor der Brutzelle vorübergehend deponiert würden.

Beide Arten der präparierten Bruthilfen wurden aus nicht erklärbaren Gründen in keinem Fall von Symmorphus crassicornis (PANZER) angenommen.

\subsection{Beobachtung des Brutverhaltens mittels eines Sichtfensters}

Bislang gelang es dem Autor noch niemals, dass Symmorphus crassicornis (PANZer) ihre Brutzellen in einem durchsichtigen Acrylrohr anlegte, so wie es THIEDE, H. (1981) für andere solitäre Hymenopteren beschreibt. Auch dann nicht, wenn normale Bambusröhren sehr sparsam ausgelegt wurden, dafür aber Acrylröhren in allen Größen und in Hülle und Fülle einer Wespe zur Verfügung

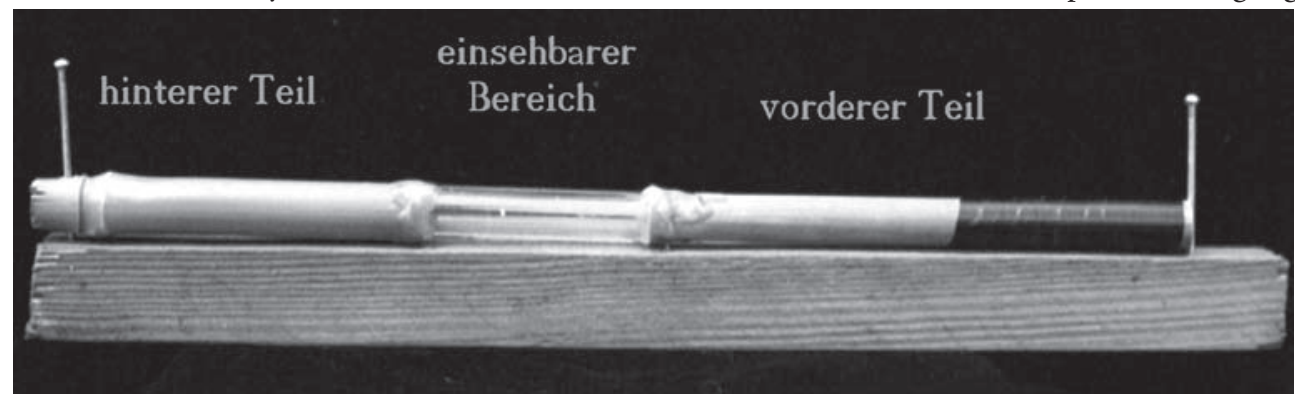

Fig. 3: Bambusrohr mit eingeklebten Teil eines Acrylrohres. Die beiden Nägel dienten lediglich vorübergehend für den Halt beim Klebevorgang.

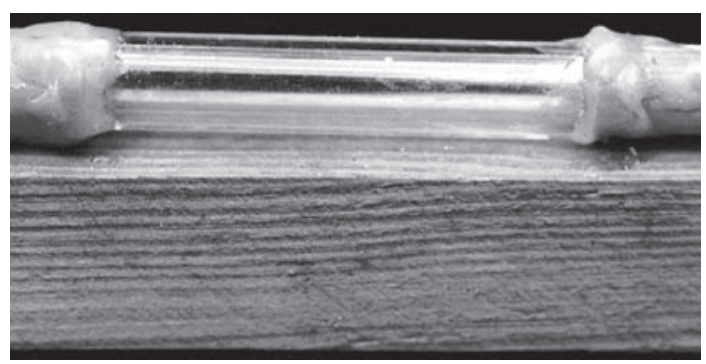

standen. Um trotzdem eine Sichtkontrolle zu ermöglichen, wurde im Jahr 2005 ein Bambusrohr in der Mitte durchgetrennt ${ }^{2}$ und an der Schnittstelle ein $5 \mathrm{~cm}$ langes Stück Acrylrohr mit gleichen freiem Durchmesser wie das Bambusrohr $(8 \mathrm{~mm})$ eingeklebt (Fig. 3).

Fig. 4: Der $5 \mathrm{~cm}$ lange Acrylteil.

\footnotetext{
${ }^{2}$ Dieser Vorschlag stammte vom Sohn des Autors (Wolf Tölke), der an den Aktivitäten seines Vaters viel Anteil nimmt.
} 
Um die Wespe nun in dieses so präparierte Rohr zu locken, wurde ein von ihr besetztes normales Bambusrohr zum Zeitpunkt des Verschließens ihrer ersten Brutzelle vom Autor gegen das präparierte Rohr ausgetauscht. Es gab zwar anfangs Irritationen im Verhalten der solitären Wespe, aber schließlich nahm die Wespe dieses Rohr an. Leider war der Autor durch diesen Erfolg zu ungeduldig, denn er filmte die Wespe mit Larve mehrmals beim Durchlauf dieses Klarsichtstückes mittels Video-Camcorder. Aber durch den starken Lichteinfall, erforderlich für das Filmen, zeigte die Wespe immer wieder Irritationen im Verhalten. Sie baute zwar ihre angefangene Brutzelle noch zu Ende, hat dann aber dieses so präparierte Rohr nicht wieder aufgesucht.

Im Jahr 2006 zog bei dem Autor, in dem gründlich gereinigten „Rohr mit Sichtbereich" wiederum eine Symmorphus crassicornis (PANZer) ein.

Den kritischen Acrylbereich hatte der Autor diesmal gleich abgedunkelt, um jegliche Störung zu vermeiden. Die Wespe legte nun Brutzelle für Brutzelle an.

Schließlich näherte sich die Wespe mit ihren Brutzellen ganz offensichtlich dem Acrylbereich. Fast während des Gesamtbrutverhaltens erfolgte die handschriftliche

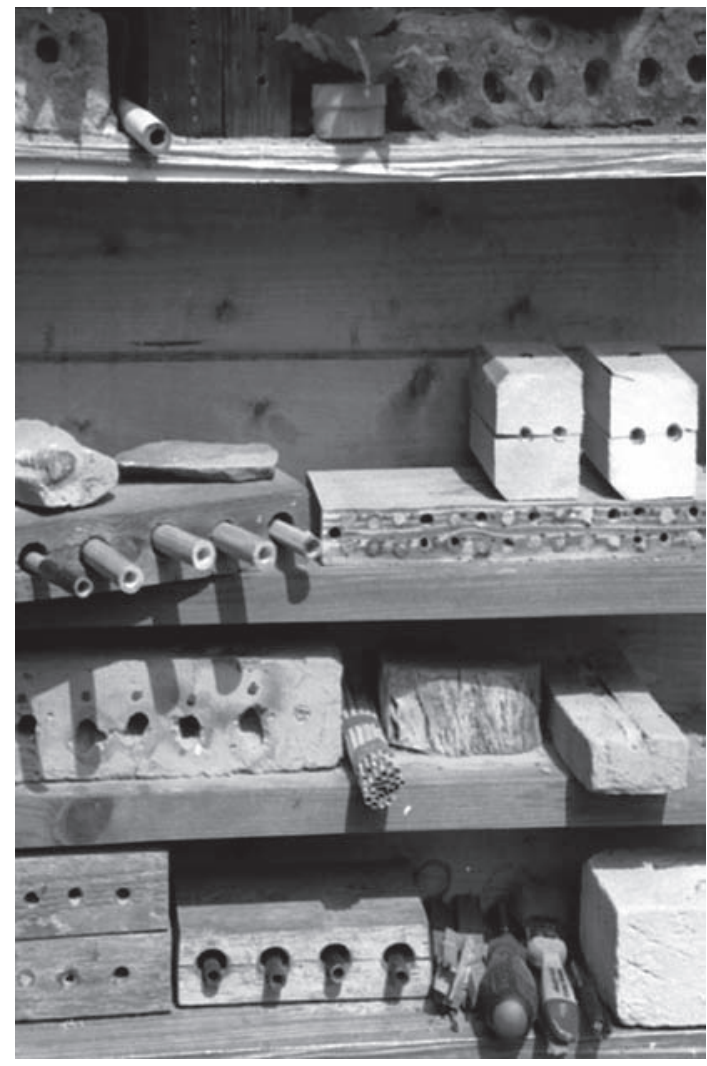

Fig. 5: Teileinblick auf die Anlage des Autors, die verschiedene Arten an Nisthilfen für solitäre Hymenopteren enthält und von diesen zum Anlegen von Brutzellen auch angenommen wurden.

Registrierung eines jeden Ein- und Ausflugs in Form der Uhrzeit Sekundengenau. Gleichzeitig wurde nach jedem Ausflug der Wespe das „Acrylfenster“ in Augenschein genommen. Auf diese Weise gelang es, genau von dem Zeitpunkt an, wo die Wespe mit ihrem Brutverhalten den Sichtbereich erreichte, eine möglichst lückenlose Beobachtung durchzuführen. Er konnte so genau feststellen, wohin die Wespe bei einem Larveneintrag die Beute ablegt. Durch gleichzeitige Zeitregistrierung war es möglich, die jeweils vorliegende Verweildauer der Wespe im Innern des Acrylbereichs nachträglich der aktuellen visuellen Beobachtung in diesem Bereich sekundengenau zuzuordnen. Auf diese Weise erfolgte genau für drei Brutzellen das Erfassen des Brutverhaltens und der exakten Werte der Wespenaktivität, ohne dass sie gestört schien. Da der Autor von Brutzelle zu Brutzelle Schritt für Schritt den Acrylbereich immer heller gestaltete, gelang es schließlich sogar, ohne das die Wespe sichtbar in ihrem Verhalten gestört reagierte, die Einlagerung der Larven durch die Wespe in der letzten Brutzelle für eine Weile direkt zu beobachten. Fotoaufnahmen wurden von der Ablage ihrer Beute nur bei ihrer ersten Deponierung im Sichtbereich des Rohres immer erst nach Verlassen des Rohres gemacht, um eine unnötige Störung zu vermeiden. Auch auf die Gefahr hin, eine Störung auszulösen, schienen zumindest diese Fotoaufnahmen notwendig zu sein, um Mindestbeweise zu erhalten. 


\section{Ergebnisse der Experimente und der Beobachtungen}

\subsection{Eingetretene Ergebnisse durch Änderung der Lageposition des Eies}

Das Ergebnis eines im Jahre 2000 groß angelegten Experiments erschien Anfangs recht enttäuschend abzulaufen, denn es kam nach der jeweiligen Lageveränderung des Brutrohres um 180 Grad und damit zur Unmöglichkeit eines Schwingens des Eies durch Fühlerschlag, nicht in einem einzigen Fall zum sofortigen Abbruch des Jagdverhaltens der Wespe und somit auch nicht zum Ende des Eintragens von weiterer Beute. Munter trug sie weitere Beute ein. Es schien so $\mathrm{zu}$ sein, wie es bei einigen Fällen in vergangener Zeit immer wieder einmal vorgekommen war. Nach vier bis fünf Larven folgte die Sortierphase und dann das ordnungsgemäße Verschließen der Brutzelle. Nachdem schließlich die Wespe auch ihr Rohr nach dem Anlegen von mehreren Brutzellen ordnungsgemäß verschlossen hatte, wurde das Bambusrohr der Länge nach vom Autor aufgeschlitzt, um so zu erkennen, wieviel Larven die Wespe jeweils in die einzelnen Brutzellen gelegt hatte. Bis auf die erste Brutzelle und die Zelle, bei der keine Drehung des Rohres erfolgt war (Fig. 6), konnte man deutlich erkennen, dass die Wespe alle Zellen nicht wie sonst üblich voll mit Larven gefüllt hatte.

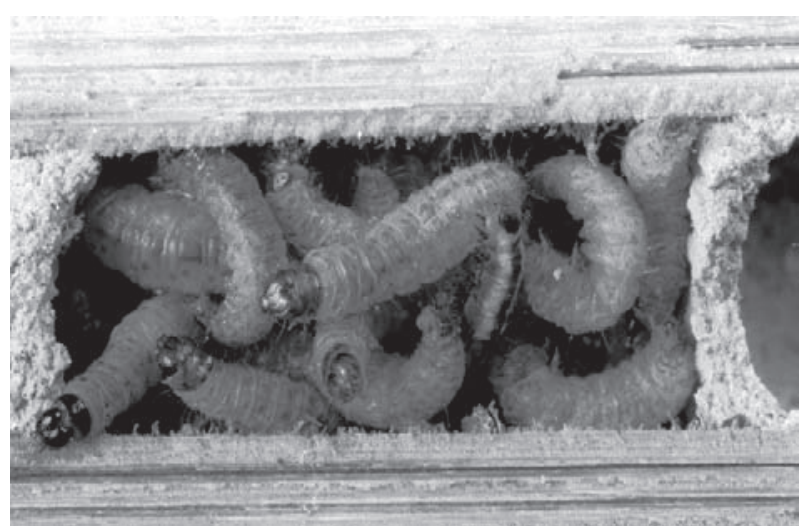

Fig. 6: Blick in das Innere einer mit Blattwespenlarven gefüllten Brutzelle. Diese Brutzelle ist normal gefüllt. Der Blick auf die Larven war möglich, weil das Bambusrohr der Länge nach aufgeschlitzt wurde.
Es verstärkte sich beim Autor der schon früher bestehende Verdacht, dass Symmorphus crassicornis (PANZER) eine gewisse Anzahl ihrer Beute nicht gleich in die jeweilige Brutzelle einsortiert, sondern erst einmal irgendwo in der Brutzelle ablegt. Also so, wie der Autor es indirekt schon in der Vergangenheit aus den registrierten Aktivitätszeiten postuliert hatte. Wenn dieser Verhaltensphase tatsächlich so wie vermutet abgelaufen war, konnte die Wespe bei dieser bloßen Ablage ihrer Beute außerhalb der Brutzelle und damit außerhalb des Eibereichs gar nicht mit dem hängendem Ei in Berührung gekommen sein.

\subsection{Beobachtungen, die durch das Sichtfenster erfolgen konnten}

Um das im Jahr 2006 beobachtete Verhalten besser verstehen zu können, wollen wir die im hinteren Teil des Bambusrohres angelegten Brutzellen als den „Bereich X“ nennen. Dies deshalb, weil ja nicht mit Sicherheit gesagt werden kann, wieviel Brutzellen hier schon angelegt waren. Sie baute die jetzt folgende Brutzelle noch nicht unmittelbar hinter dem Acrylbereich. Sie ließ zwischen Sichtbereich und Brutzelle „X+1“ noch so viel Platz, der den Bereich einer weiteren Brutzelle entsprochen hätte. Also zwischen der Brutzelle und dem Bereich der Ablage bestand noch ein zusätzlicher freier Raum. Deutlich war zu erkennen, dass die Wespe ihre erste Larve im Acrylbereich ablegte. Also weit ab von ihrer geplanten Brutzelle. Dann folgte die Einbringung und die Ablage der Larve Nr. 2 (Fig. 7). 
Bei der vom Autor nach dem Ausflug der Wespe durchgeführten Sichtkontrolle, musste er aber feststellen, dass die Larve Nr. 1 nun aus dem Acrylbereich verschwunden war. Mit sehr großer Wahrscheinlichkeit konnte sie nur von der Wespe in die Brutzelle reingezogen worden sein. Interessant, dass für das Ablegen der Larve Nr. 2 und das vermutliche „einsortieren " der Larve Nr. 1 insgesamt 87 Sekunden benötigt wurden, also bedeutend mehr Zeit als bisher für andere Messungen bekannt war. Danach brachte sie eine relativ große Larve Nr. 3. Sie wurde vom Eingang aus gesehen vor Larve Nr. 2 abgelegt (Fig. 8).

Jetzt folgte das bloße Ablegen einer noch größeren Larve Nr. 4. Sie kommt noch weiter nach vorn Richtung Ausgang zur Ablage. Es folgten Larve Nr. 5 und Nr. 6, aber als Besonderheit im Verhalten, die vermutlich von der Wespe schon in die Brutzelle einsortierte Larve Nr. 1 lag jetzt auch wieder im Acrylsichtbereich, also befanden sich dort jetzt alle 6 bisher gefangenen Larven. Was nun geschah war im Verhaltensablauf ganz erstaunlich. Nachdem sie Larve Nr. 6 abgelegt hatte, begann sie noch in der gleichen Zeitphase mit dem Einsortieren der Beute in die Brutzelle im wahrsten Sinne des Wortes. Denn ganz außergewöhnlich war, nicht die der Brutzelle am nächsten liegende Larve wurde zuerst in die Brutzelle gezogen, sondern die größte gefangene Larve, Blattwespenlarve Nr. 4. Dann brachte sie die zweitgrößte Beute

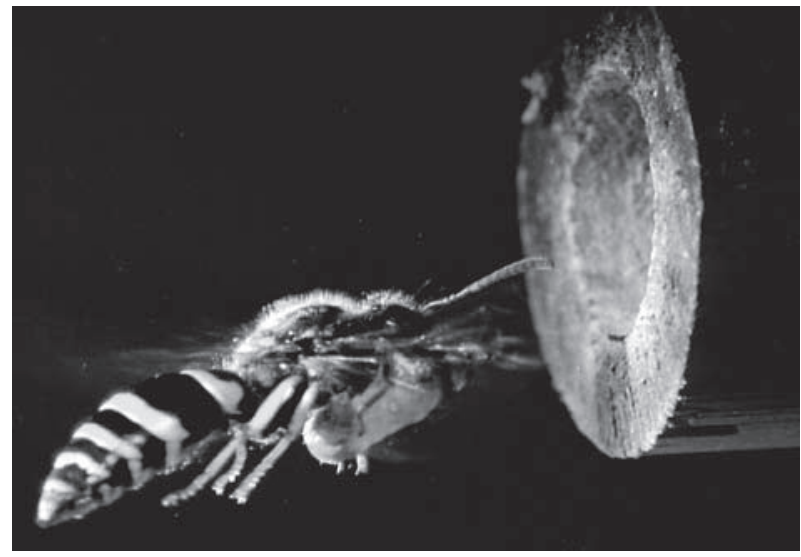

Fig. 7: Eine Larve wurde erbeutet. Bei größerer Beute kann man die Larve beim Einflug durchaus erkennen.

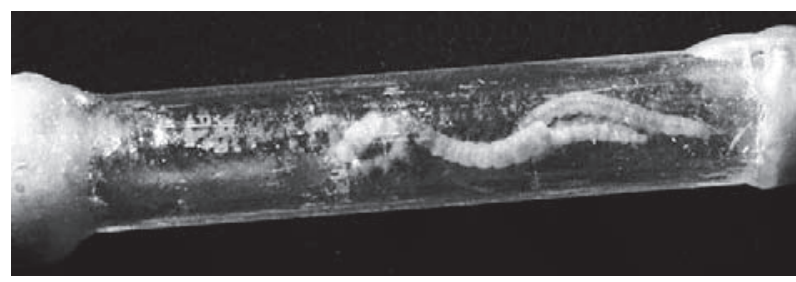

Fig. 8: Die im Acrylbereich vor der Brutzelle vorerst abgelegten Larven kann man bei einer Zwischen-Beobachtung zweifelsfrei erkennen.

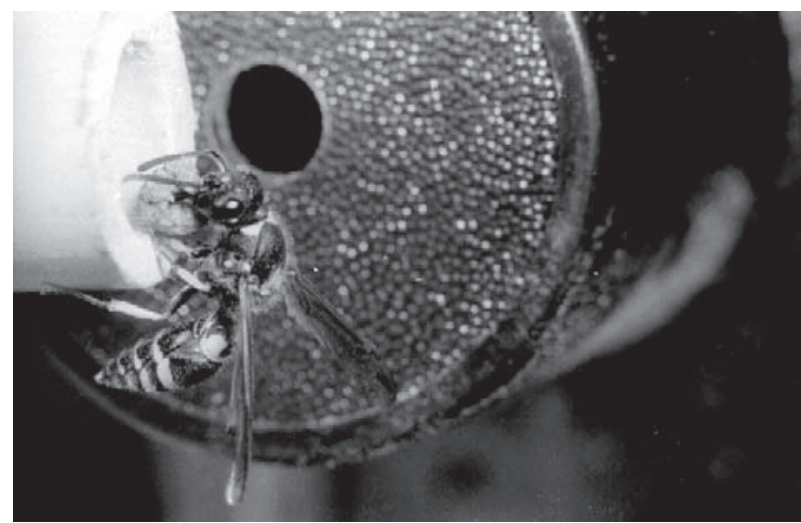

Fig. 9: Eine Wespe trägt Lehm in die Brutzelle, um diese zu Verschließen. Das im Hintergrund befindliche schwarze Gebilde ist ein Lichtschrankenempfänger, der für eine vollautomatische Bildauslösung sorgt.

also Larve Nr. 3 in die Brutzelle. Schließlich folgten die kleineren Larven. Nachdem alle Larven aus dem Sichtbereich verschwunden waren, holte sie Lehm von einem in der Anlage deponierten Lehmstein als Material zum Verschließen ihrer Brutzelle (Fig. 9). 
An anderer Stellewurde schon erwähnt, dass eine Wespe manchmal in ihrem zeitlichen Brutverhalten in bestimmter Weise von ihrem sonst gewohnten „Standardzeitprogramm“ abweicht, sie also gewissermaßen eine „persönliche Note“ praktiziert. Diese im Jahre 2006 hier Brutzellen anlegende Wespe zeigte in ihrem Zeitverhalten sogar eine ganz ungewohnte Besonderheit. Immer dann, wenn eine Larve, manchmal aber auch erst, wenn sie zwei ihrer abgelegten Larven in die Brutzelle einsortiert hatte, verließ sie für kurze Zeit die Brutröhre. Dann setzte sie aber das Einsortieren innerhalb des Rohres fort, danach folgte das Verschließen dieser Brutzelle.

Als der Autor am Abend eines Regentages die Beobachtung wieder aufnahm, entdeckte er im Acrylbereich eine kleine graue Larve (Larve Nr. 1). Da die Wespe zu diesem Zeitpunkt keine Aktivität erkennen ließ (es ist $18 \mathrm{Uhr}$ 30) unterbrach er die Beobachtung. Bei der erneuten Sichtkontrolle um 19 Uhr 45, bemerkte er, dass die Larve Nr. 1 verschwunden war. Die Wespe ragte nur mit Ihrem Abdomen aus dem Bambusbereich heraus und arbeitete irgendwie intensiv. Darin wurde indirekt erkennbar, dass sie jetzt die Brutzelle Nr. „X+2“ anlegte. Diese Zelle müsste nach dem Verhalten der Wespe zu urteilen innerhalb des hinteren Bambusbereich und hier direkt vor dem Acrylbereich angelegt sein.

Die Sichtkontrolle ergab, dass im Acrylbereich direkt vor dem Bambusteil zwei mittelgroße Larve lagen, also Larve Nr. 2 und 3. Dann kam die Wespe schließlich mit einer sehr großen Larve angeflogen. Für das Ablegen dieser Larve Nr. 4 benötigte sie 51 Sekunden. Die Ablage geschah direkt nachgeordnet zu den beiden mittelgroßen Larven, also in Richtung Ausgang. Dann folgte die Larve Nr. 5, für deren Einlagerung sie eine sehr lange Zeit in Anspruch nahm. Nach einem 8 Sekunden dauernden Ausflug ein erneuter Einflug. Um zu erkunden, was hier geschah, erfolgte vorsichtig eine Sichtkontrolle. Die Wespe war gerade dabei, die mittelgroße Larve (also Larve Nr. 2) in die Brutzelle zu ziehen. Für nur vier Sekunden flog sie erneut nach draußen. Wiederum vorsichtig eine Sichtkontrolle. Die Wespe ragte nur noch mit dem Abdomen aus der Brutzelle heraus. Im Sichtbereich lagen nur noch zwei Larven. Wiederum verließ sie das Brutrohr, aber nur für 2 Sekunden und nur soweit, dass sie in der Öffnung wenden konnte. Nach 22 Sekunden flog sie endgültig aus. Der Acrylsichtbereich war nun leer. Nach mehreren Ein- und Ausflügen kam sie schließlich nach 15 Minuten mit einer Larve angeflogen. Die Kontrolle ergab, dass sich diese Larve im Sichtbereich befand. Die Wespe dagegen steckt mit ihrem Kopf in der Brutzelle und arbeitet dort intensiv. Erst nach 12 Minuten (!) verlässt sie das Rohr. Ob sie nach dem erneutem erfolgreichen Jagdausflug für die Einsortierung der Larve Nr. 6 alle schon einsortierten Larven wiederum vorübergehend im Vorraum auslegte, kann nicht ausgesagt werden, weil eine zwischenzeitliche Sichtkontrolle (leider, wie sich später herausstellte) unterlassen blieb. Nach dem letzten Ausflug fliegt sie zum Lehmstein innerhalb der Anlage. Sie verschließt nun den hinteren Bambusrohrteil.

Der nächste Tag ergibt bei den Kontrollen das gewohnte Bild. Im eigentlichen Zellbereich unmittelbar vor der Trennwand zur Brutzelle $\mathrm{X}+2$ hin, liegt zuerst nur eine winzig kleine Larve. Eine Stunde später folgt eine weitere kleine Larve (Larve Nr. 2), die unmittelbar neben Larve Nr. 1 abgelegt wird. Danach bringt sie eine etwas größere Larve (Larve Nr. 3). Alle drei liegen, nachdem die Wespe das Rohr verlassen hat, nebeneinander direkt vor der Lehmwand der Brutzelle X+2. Nach einem erneuten Einflug und einem nach 4 Minuten erfolgenden Ausflug, sind alle drei Larven weiter Richtung Ausgang platziert. Es ist jetzt gewissermaßen der eigentliche Brutzellbereich (Brutzelle X+3) frei. Jetzt trägt sie eine große Larve (Larve Nr. 4) ein und legt sie vor den schon deponierten Larven in Richtung Ausgang ab. Es folgt noch die Beute Nr. 5. Sie wird neben Larve Nr. 4 deponiert. Die Wespe bringt eine weitere große Larve (Larve Nr. 6). Sie deponiert sie vor Larve Nr. 5, sie benötigt für diese Deponierung nur 27 Sekunden. Nun folgt Larve Nr. 7, die vor Nr. 6 ihren Platz findet. 40 Sekunden braucht die Wespe nun zum Ablegen. 
Aber diese Beute liegt schon außerhalb des Acrylbereichs somit im vorderen Bambusrohrteil. Die nächste Larve Nr. 8 ist eine sehr große Blattwespenlarve. Sie findet ihren Platz im vorderen Bambusteil. Nach 19 Minuten hat sie alle Larven in den Brutzellenbereich gebracht. Aber sie ist offensichtlich mit dem Einlagerungsergebnis nicht zufrieden, denn sie zieht immer wieder einzelne Larven aus dem Zellbereich heraus und versucht sie in der Brutzelle besser zu ordnen. Diese Umlagerung dauert 19 Minuten (!). Leider ist das an der Decke hängende Ei nicht zu erkennen auf Grund der Trübung im Acrylstück. Da sie die Einlagerung aber bis fast zur Decke ausführt, scheint ein Pendelschlag nicht mehr möglich zu sein. Nach dieser Umlagerung verschließt sie die Brutzelle mit Lehm.

Nachdem diese Brutzelle ordnungsgemäß verschlossen war, baute die Wespe genau an der Grenze zwischen Acrylteil und vorderem Bambusteil eine weitere Lehmwand. Am nächsten Tag verschloss sie das Rohr endgültig mit einem weiteren Verschluss.

\section{Diskussion}

In der Diskussion sollen drei Hauptfragen im Mittelpunkt der Betrachtung stehen. Diese sind:

- Welche Variationsbreite kann im Verhalten bei dem Eintragen und Lagern der Beute auftreten?

- Wie ermittelt die Wespe die Losgrößen für erbeuteten Larven, die sie vorübergehend im Vorraum der Brutzelle ablegt?

- Warum sortiert die Wespe die erbeuteten Larven der Größe nach in der Brutzelle?

\subsection{Welche Variationsbreite kann im Verhalten bei dem Eintragen und Lagern der Beute auftreten?}

Es besteht kein Zweifel daran, dass Symmorphus crassicornis (PANZER) ihre Beute fast nie gleich beim Einbringen in die aktuelle Brutzelle einsortiert. Und wenn sie doch einmal im Anfangsstadium eine Larve gleich in die Brutzelle einlagert, holt sie diese mit ziemlicher Sicherheit nach einiger Zeit wieder heraus, um sie zu den anderen Larven im Vorraum der Brutzelle zu legen. Nur im Endstadium der Einlagerung kommt es vor, dass sie hin und wieder eine Einzellarve sofort in die Brutzelle einsortiert.

Somit besteht ihr Grundverhalten darin, ihre erbeuteten Larven jeweils einzeln vorerst einmal vor dem Brutzellenbereich abzulegen. Dabei sorgt sie dafür, dass keine der Larven oberhalb einer schon deponierten Larve zu liegen kommt. Also sie legt sie nie übereinander. Bei dem bloßen Ablegen der Beute treten immer relativ kurze Zeiten auf.

Diese Aussage kann deshalb mit ziemlicher Sicherheit so getroffen werden, weil es dem Autor gelang, bei der Analyse einen neuartigen methodischen Ansatz anzuwenden. Dieser besteht in der Erfassung der sekundengenauen Zeitregistrierung beim Eintragen der Beute, bei gleichzeitigem Registrieren der zugehörigen Ausflugzeit. Diese so ermittelten Aufenthaltszeiten der Wespe in der Brutröhre werden der gleichzeitigen direkten Beobachtung des Geschehens in der Brutröhre mittels Acrylfenster zugeordnet. Dabei wurde erkennbar, dass das Verhalten mit hoher Wahrscheinlichkeit komplex abläuft und so gestaltet ist, dass es in bestimmten Situationen durchaus etwas von dem eigentlichen Grundverhalten abweichen kann. Folgende Variationsbreite im Verhalten kann eintreten: 
- Die Wespe lagert manchmal die im Vorraum der Brutzelle abgelegten Larven um. Sie bringt sie aber hierbei noch nicht in die eigentliche Brutzelle. Dieses Verhalten steht möglicherweise im Zusammenhang mit der späteren Einsortierung der Larven der Größe nach.

- Die Wespe holt manchmal eine oder auch hin und wieder zwei schon in der Brutzelle einsortierten Larven wieder heraus und legt sie in den Vorraum zu den dort schon lagernden Larven ab. Es spricht viel dafür, dass mit einem solchen ungewöhnlichen Verhalten sich die Wespe die Möglichkeit einer späteren Sortierung der Größe nach aller erbeuteten Larven verschafft.

- Nachdem die Wespe in nacheinander ablaufenden Verhaltensphasen mehrere Blattwespenlarven lediglich im Vorraum der Brutzelle, jeweils einzeln, abgelegt hat, beginnt sie, nachdem sie ihre letzte Larve dieses Loses in den Vorraum eingetragen hat, ohne eine bisher erkennbare Zwischen-Verhaltensphase mit dem Einsortieren aller im Vorraum liegenden Larven (Beginn der 1. Sortierphase).

- Bei dem Einsortieren der Blattwespenlarven in die Brutzelle verfährt sie der Größe nach.

- Liegt schon eine bestimmte Mindestmenge an Beute einsortiert in der Brutzelle, kommt es hin und wieder vor, dass sie noch einmal ausfliegt und mit einer Larve nach einer gewissen Zeit angeflogen kommt. Diese so eingebrachte Beute sortiert sie aber sofort in die Brutzelle ein. Manchmal fängt sie in dieser Situation auch mehrere Larven hintereinander und legt diese aber wiederum erst einmal im Vorraum ab.

- Auffällig war bei einem Einzeleintrag einer Larve und deren Einsortierung in die Brutzelle, dass die Wespe ganz offensichtlich bemüht ist, die jeweils angeschleppte Larve nicht unterhalb sondern vielmehr seitlich verschoben unter dem hängendem Ei einzulagern. Eine dann noch zweite folgende Beute sortierte sie auf der gegenüberliegende Seite unterhalb des Eies ein. Dadurch entstand unterhalb des frei schwingenden Eies ein deutlich erkennbarer freier Raum.

Alle die hier erläuterten unterschiedlich möglichen Verhalten innerhalb des Brutgeschehens sollten nie isoliert betrachtet werden. Denn alles ist miteinander verzahnt. Dadurch erhält das Brutverhalten ganz offensichtlich eine Komplexität, mit der die Wespe in die Lage versetzt wird, sich bei verschiedenen Situationen während der Ablage oder der Einsortierung von Larven den Erfordernissen in Bezug auf Menge der Larven jederzeit eine optimale Lösung zu finden. Diese hier postulierte Komplexität böte ihr gleichzeitig die Möglichkeit, jederzeit erfolgreich ihre Brutfürsorge (vielleicht besser Brutvorsorge) zu erfüllen. Durch eine ganz gezielte Aktivität während der Einsortierung startet sie einen Vorgang, der für sie letzten Endes ein positives oder negatives Signal liefert. Sie ist dadurch in der Lage, die eingelagerte optimale Larvenmenge mit einer einfachen Methode zu erreichen. Dieses Signal erhält sie nach dem Schlag gegen das frei schwingende Ei, durch die Rückschwingung des Eies, das von dem gleichen Fühler abgefangen wird. Durch dieses Signal kann sie mit ziemlicher Sicherheit jederzeit entsprechend handeln. Im positiven Sinne geschieht dies immer dann, wenn noch Raum in der Brutzelle unterhalb des Eies für eine zusätzliche Einlagerung einer oder mehrerer Larven bestehen sollte. Dadurch ist sie in der Lage, im Bedarfsfalle in das eigentlich schon abgeschlossene Jagdverhalten zurück zu schalten. Demnach kommt diesem Faden, an dem das Ei hängt, eine ganz zentrale Bedeutung im gesamten Verhaltensablauf der Beuteeinsortierung zu. Der vom Fühler aufgefangene Rückschlag des Eies, deren Aufpralldruck ganz sicher unterschiedlich sein könnte, besitzt als Signal vermutlich sogar eine quantitative Aussage. Die Wespe besäße dann sogar eine sehr feine Justierung, mit der sie vielleicht unterscheiden kann, wie groß der Bedarf an weiteren Larven noch wäre. Denn es 
kommt sowohl vor, dass noch mehrere Larven gefangen werden oder im Bedarfsfalle auch nur eine einzige, weil im letztgenannten Falle das Signal der Wespe indirekt „mitteilt“, mehr Platz als für eine Larve ist nicht vorhanden.

Das Jagdverhalten ist für die Wespe dagegen für diese aktuelle Brutzelle dann beendet, wenn sie dieses Signal nicht mehr erhält. Sie verschließt dann die Zelle mit Lehm. In der Regeltechnik entspräche diesem Signal der Schalter „EIN“ oder „AUS“. Eine solche Zuordnung würde dem Wirkungsgefüge einer Verhaltensleistung entsprechen, wie sie von den nachfolgenden Autoren beschrieben wurde, allerdings für andere Arten (Mittelstaedt, H. 1961; Todt, D. J. 1968).

\subsection{Wie erkennt die Wespe die Losgröße der zu erbeuteten Larven?}

Aus alle dem bisher Gesagten verschiebt sich zwangsläufig die jetzt aktuelle Fragestellung in Richtung der Losgröße der im Vorraum vorübergehend abgelegten Larven. Wie erkennt die Wespe in ihrem Standardgrundprogramm, dass in dem einen Fall die gesamte Jagdphase mit dem Erbeuten von 4 bis 6 Larven gestartet werden muss und in einem anderen Fall lediglich nur noch eine einzige Larve erforderlich ist? Im erstgenannten Fall ergibt sich die Frage wie sie die Strecke für das Ablegen und somit für den Umfang der dort abgelegten Beute steuert. Wie schon erwähnt, beendet sie ihr Jagdverhalten nach einer bestimmten Anzahl eingetragener Beute und beginnt dann mit dem Einsortieren in die Brutzelle. Welches ist bei dieser Voreinlagerung die beendende Bedingung für sie? Schreitet sie diese Larvenstrecke ab, um festzustellen, ob die Anzahl an Larven schon ausreicht? Auffällig auf jeden Fall ist, dass hin und wieder auch beim bloßen Ablegen einer Larve etwas längere Zeit auftritt, ohne dass sie an der Lage der Larven irgend etwas veränderte. Dieses einmal angenommene Messen der Strecke würde demnach aber nicht nach jedem Eintrag einer Larve erfolgen. Denn sonst ließen sich die immer wieder sehr kurzen Verweilzeiten nicht erklären. Aber wie sollte dieses Messen erfolgen?

\subsection{Warum sortiert die Wespe die erbeuteten Larven der Größe nach in der Brutzelle?}

Die vorläufige Ablage im Vorraum der Brutzelle geschieht so, wie sie sich aus der Reihenfolge des Eintragens ergibt. Liegt eine bestimmte Menge an Beute aufgereiht unmittelbar hintereinander vor der Brutzelle, zieht die Wespe schließlich Larve für Larve in die Brutzelle. Aber nicht der Reihe nach, wie sie im Rohr liegen, sondern, und dies ist das erstaunliche, der Größe nach. Die größte Larve zuerst, dann folgt die zweitgrößte und so weiter.

Dass die Wespe die Beute der Größe nach aus den im „Vorraum“ zur Brutzelle „aufgereiht liegenden Larven" herausfinden kann, wäre ja noch vorstellbar. Ihre Larven liegen ja alle schön aufgereiht hintereinander und nie übereinander. Eine solche Art der Ablage ist sicher kein Zufall, denn die Sortierung muss ja etwas später reibungslos und zeitlich möglichst schnell zu erledigen sein. Auffällig daran, dass alle Larven in dieser ersten Verhaltensphase, also der bloßen Ablage, immer völlig frei liegen, auch dann, wenn einmal zwei kleinere nebeneinander liegen. Sicher ist nun aber auf jeden Fall, dass die Wespe immer dann, wenn es nur um eine Ablage ihrer Beute im Vorraum geht, kein Fühlerkontakt mit dem Ei erfolgt. Einfach deshalb nicht, weil sie das hängende Ei aus dieser Position nicht erreichen kann. Die Losgröße der gefangenen Larven muss also von anderen Auslösebedingungen gesteuert werden. Ist eine solch bestimmte Menge erreicht, zieht sie die Larven in die Brutzelle. Dabei passiert dann jedesmal der schon erwähnte Fühlerschlag gegen das am Faden hängende Ei. Unabhängig davon, ob der Schlag gegen das Ei und der folgende Pendelrückschlag noch kommen kann oder nicht, die Gesamtheit der im Vorraum gesammelten Beute wird auf jeden Fall vollständig in die Brutzelle eingelagert. 
Aber wie gestaltet sie die Einsortierung der Größe nach, wenn sie noch nachträglich einzelne gefangene Larven einsortieren will (z. B. Larve Nr. 6 in Brutzelle X+2)? Zieht sie dazu noch einmal alle Larven aus der Brutzelle wieder heraus? Auffällig ist auf jeden Fall die sehr lange Verweildauer (12 Minuten!), die sie für diese Einsortierung in Anspruch nahm.

Durch die bisher getroffenen Aussagen wird auch auf einmal verständlich, warum die Wespe in dem unter Punkt 2.1 beschriebenen mehrfachen Experiment die Zelle nicht voll mit Larven versorgt. Ihre 4 bis 6 gefangenen Larven lagen zuerst einmal ja außerhalb des Eibereichs. Und eine Einlagerung erfolgte somit unabhängig davon, ob ein Kontakt mit dem Ei oder genauer gesagt ein Pendelschlag gegen das Ei möglich war. Sie sortierte eben alles in die Brutzelle ein, was sie gefangen hatte. Ganz offensichtlich liegt hier ein geschlossenes Verhaltenssyndrom vor (siehe hierzu Темввоск, G. 1955). Aber da sie ein Signal von dem pendelnden oder nichtpendelnden Ei nicht erhalten kann, weil es am Boden liegt, fliegt sie nicht mehr zum weiteren Beutefang aus.

Das erst einmal bloße Ablegen der Beute bringt ohne Zweifel auch ökonomische Vorteile, kann sie doch so jedesmal schnell wieder zur Jagd ausfliegen.

Aber warum sortiert sie ihre Beute überhaupt der Größe nach? Gestaltet sich die gesamte Einsortierung dadurch einfacher? Aber vieles spricht für einen anderen Grund. Auch nach der Betäubung der gefangenen Larven durch die Wespe führen diese noch zuckende Bewegungen aus. Die können bei großen Larven unter Umständen recht kräftig ausfallen. Da die kleine Eilarve der Wespe von der Decke in die unter ihr einsortierte Beute fällt, bestände natürlich durchaus die Gefahr, dass sie durch eine solche kräftige zuckende Bewegung einer größeren Larve beschädigt werden könnte ${ }^{3}$. Diese mögliche Gefahr bestände nicht in diesem Umfang, wenn die Wespenlarve auf eine relativ kleine Blattwespenlarve fällt. Da sie durch Aussaugen der ersten Larve sehr schnell an Größe gewinnt, würde dann die Gefahr für sie immer kleiner werden.

In Betracht gezogen werden müsste auch die Überlegung, dass für die relativ kleine Eilarve als erstes Futter kleinere Larven zuträglicher sein könnten ${ }^{4}$. Auf jeden Fall muss die Einsortierung der Larven in die eigentliche Brutzelle nach ihrer Größe für die Wespe sehr bedeutsam sein. Ist doch ein Großteil des Brutverhaltens auf dieses außergewöhnliche Verhalten abgestimmt.

Unabhängig von all dem gesagten und bewiesenen Verhalten bleiben viele weitere Fragen für bestimmte Verhaltensabläufe noch völlig ungeklärt. Diese sollten in Zukunft bei ähnlichen Untersuchungen stärker beachtet werden. Ihre endgültige Klärung gelänge aber nur dann, wenn man erreicht, wiederum einmal eine solitäre Wespe dieser Art in eine entsprechend präparierte Bambusröhre zu locken. Aber durch die vorliegenden Erkenntnisse ist nun bekannt, auf welches Verhalten in der Brutröhre man dann in der Zukunft gezielt achten müsste. Auch sollte man bei all diesen hier beschriebenen Erfolgen bedenken, dass ein Großteil dieser Ergebnisse erst für ein einziges Individuum dieser Art vorliegt. Vorsicht für endgültige Aussagen ist auch deshalb geboten, weil diese Wespenart zu individuellem Verhalten neigt.

\section{Literatur}

Mittelstaedt, H. 1961: Die Regelungstheorie als methodisches Werkzeug der Verhaltensanalyse. - Naturwissenschaften 48: 246-254.

Темввоск, G. 1955: Zur Ontogenese einiger Verhaltenssyndrome beim Rotfuchs. 1. Arbeitstagung über zentrale Regulation der Funktion des Organismus, Leipzig.

\footnotetext{
${ }^{3}$ Diesen Gedanken äußerte als erste Ingeborg Tölke.

${ }^{4}$ Diesen Gedanken äußerte in einem Gespräch Dr. E. Fendt.
} 
Thiede, U. 1981: Über die Verwendung von Acrylglasröhrchen zur Untersuchung der Biologie und Ökologie solitärer aculeater Hymenopteren (Hymenoptera). - Deutsche Entomologische Zeitschrift 28: 45-53.

Tорт, D. J. 1968: Zur Steuerung unregelmäßiger Verhaltensabläufe. Sonderdruck aus Kybernetik. 465-485. Oldenburg und München.

TöLKE, A. 1966: Zeitregistrierungen und Experimente an Brutröhren der Lehmwespe Symmorphus crassicornis (Panzer) zur Analyse ihres Verhaltens. Nicht veröffentlichte Diplomarbeit.

Tölke, A. 1968: Weitergehende wissenschaftliche Erkenntnisse durch gezielten Einsatz des 16 mm Films, demonstriert am Beispiel von Verhaltensanalysen solitärer Hymenopterae. Vortrag auf 1. ICREC, Brno 1968, veröffentlicht 1969; 166-180.

TöLke, A. 1973: Quantitative und qualitative Analyse von Verhaltenssequenzen mit Hilfe einer elektronischen Kleinrechenanlage - demonstriert am Beispiel Osmia rufa (L.). - Biologisches Zentralblatt 92 (1): $1-25$.

Tölke, A. 1994: Computer-Sofortanalyse des Zeitverhaltens von Wildbienen. - In: HedtKe, C. (Hrsg.): Wildbienen. - Tagungsband der Wildbienentagung des Länderinstituts für Bienenkunde Hohen Neuendorf: 60-69.

Tölke, A. 1996: Steuerung der Beutemenge bei Symmorphus crassicornis (PANZer) (Vespidae: Eumeninae) mittels „Eikontakt“. - Bembix, Nr. 6: 9-13.

Tölke, A. 2005: Mit dem PC das Zeitverhalten einiger solitärer Hymenopteren beim Anlegen von Brutzellen Interpretieren - ein methodischer Ansatz zur Untersuchung des Verhaltens von Osmia rufa (L.), Anthophora acervorum (L.) und Symmorphus crassicornis (PANZ.). - Mitteilungen der Arbeitsgemeinschaft westfälischer Entomologen, 21 (Beiheft 12): 1-12.

\section{Anschrift des Verfassers:}

Dr. Arnim TölKe

Am Vogelsang 2b

D - 15366 Neuenhagen

Tel. 03342/7250

toelke@makro-foto.net

www.makro-foto.net

\section{Subject editor:}

Prof. Dr. J. OehlKe 